\title{
Comparative Changes of Lipid Levels in Treatment-Naive, HIV-1-Infected Adults Treated with Dolutegravir vs. Efavirenz, Raltegravir, and Ritonavir-Boosted Darunavir-Based Regimens Over 48 Weeks
}

\author{
Romina Quercia · Jeremy Roberts • \\ Louise Martin-Carpenter $\cdot$ Carlos Zala
}

Published online: 31 January 2015

(C) The Author(s) 2015. This article is published with open access at Springerlink.com

\begin{abstract}
Background and Objectives Long-term use of antiretroviral therapy (ART) to treat HIV infection has been associated with dyslipidemia and metabolic and cardiovascular complications. Available options for patients at risk of cardiovascular disease include antiretroviral drugs with improved lipid profiles. Dolutegravir is one of a new generation of HIV integrase inhibitors recently incorporated into the US Department of Health and Human Services, German, Spanish, and Italian HIV treatment guidelines as a preferred first-line third agent in combination with dual nucleoside reverse transcriptase inhibitor (NRTI) backbone therapies. To understand the lipid profile of dolutegravir in the context of combination ART, we analyzed the lipid outcomes at 48 weeks in ART-naive participants in four phase IIb-IIIb clinical trials.

Methods Variables included in this analysis were total cholesterol (TC), low-density lipoprotein (LDL) cholesterol (LDL-C), high-density lipoprotein (HDL) cholesterol (HDL-C), TC/HDL ratio, and triglycerides at baseline and week 48.

Results In a comparative analysis, dolutegravir demonstrated a broadly neutral effect on lipids versus efavirenz or
\end{abstract}

R. Quercia

ViiV Healthcare, Brentford, UK

J. Roberts

GlaxoSmithKline, Mississauga, ON, Canada

L. Martin-Carpenter

ViiV Healthcare, Research Triangle Park, NC, USA

C. Zala $(\bowtie)$

Infectious Diseases Unit, Hospital Central de San Isidro,

Buenos Aires, Argentina

e-mail: zalacarlos@gmail.com ritonavir-boosted darunavir; in both comparisons, patients taking dolutegravir exhibited smaller increases in TC, LDL-C, and triglyceride levels. In comparison with raltegravir, dolutegravir exhibited a similar lipid profile, including small increases in TC, LDL-C, and triglyceride levels for both agents. In the pooled dolutegravir analysis, minimal increases in LDL-C and triglycerides were observed but mean values at 48 weeks remained below National Cholesterol Education Program target levels. HDL-C levels increased at 48 weeks, and the mean TC/ HDL-C ratio was 0.6 at 48 weeks; these values are associated with a lower risk of cardiovascular disease.

Conclusions Together, these data show that dolutegravir has a safer lipid profile in combination ART and provides an important treatment option for older patients who may have other risk factors for metabolic syndrome or cardiovascular disease.

\section{Key Points}

The efficacy of dolutegravir has been demonstrated in randomized studies in integrase inhibitor-naive adult populations.

To date, a detailed review of the dolutegravir lipid profile has not been provided in the literature.

Dolutegravir has shown a broadly neutral effect on serum lipids in comparative clinical trials.

\section{Introduction}

Long-term use of antiretroviral therapy (ART) is related to metabolic (dyslipidemia, insulin resistance, and diabetes) 
and cardiovascular complications [1,2]. ART can induce raised levels of total cholesterol (TC), low-density lipoprotein cholesterol (LDL-C), and triglycerides (TG), as well as variable effects on high-density lipoprotein cholesterol (HDL-C) levels [3]. In a large cross-sectional study, the prevalence of hypercholesterolemia ( $>240 \mathrm{mg}$ / $\mathrm{dL})$, hypertriglyceridemia ( $>200 \mathrm{mg} / \mathrm{dL})$, and low HDL-C $(<35 \mathrm{mg} / \mathrm{dL})$ was $10-27,23-40$, and $19-27 \%$, respectively, depending on the antiretroviral regimen [4].

The treatment options available for the management of dyslipidemia in HIV infection are similar to those in the general population, with an additional option of starting therapy or switching to a different antiretroviral class with a neutral lipid profile [2]. Given the increasing number of people living with HIV infection for longer periods of time, the lipid profile of new antiretroviral agents is a critical selection factor when choosing among different options for a treatment regimen.

Dolutegravir is an unboosted integrase strand transfer inhibitor (INSTI) recently approved for combination therapy with other antiretroviral agents in HIV-1-infected subjects. Two phase III trials of dolutegravir $50 \mathrm{mg}$ once daily, in combination with fixed-dose dual nucleoside reverse transcriptase inhibitors (NRTIs), have demonstrated non-inferiority to raltegravir-based regimens [5-7]; one phase III study showed superiority to efavirenz/tenofovir disoproxil fumarate (tenofovir)/emtricitabine in ART-naive subjects [8]. Additionally, a phase IIIb study demonstrated superiority of dolutegravir to ritonavirboosted darunavir when both were used in combination with fixed-dose dual NRTI combinations [9]. As a result, the US Department of Health and Human Services (DHHS), German, Spanish, and Italian HIV treatment guidelines have been updated to include dolutegravir as a preferred first-line third agent in combination with preferred fixed-dose, dual-combination (FDC) backbones, abacavir/lamivudine, or tenofovir disoproxil fumarate/emtricitabine [10].

To highlight the lipid profile of dolutegravir $50 \mathrm{mg}$ once daily in the context of combined ART, we undertook the present analysis to describe lipid outcomes at 48 weeks in ART-naive subjects enrolled in the four phase IIb-IIIb clinical trials $[6,8,9,11]$.

\section{Methods}

Detailed methodologies for SPRING-1 (ClinicalTrials.gov identifier: NCT00951015), SPRING-2 (ClinicalTrials.gov identifier: NCT01227824), SINGLE (ClinicalTrials.gov identifier: NCT01263015), and FLAMINGO (ClinicalTrials.gov identifier: NCT01449929) have been reported previously $[5-9,11]$. In brief, SPRING-1, a phase II dose- ranging study, randomized (1:1:1:1) 205 treatment-naive adults to receive 10,25 , or $50 \mathrm{mg}$ dolutegravir or $600 \mathrm{mg}$ efavirenz, respectively, in combination with either tenofovir/emtricitabine or abacavir/lamivudine. SPRING-2 randomized $822 \mathrm{HIV}$-infected, ART-naive adults to treatment with raltegravir $400 \mathrm{mg}$ twice daily or dolutegravir $50 \mathrm{mg}$ once daily, each with either abacavir/lamivudine $600 / 300 \mathrm{mg}$ once daily or tenofovir/emtricitabine $600 / 300 \mathrm{mg}$ once daily as selected by the investigator. SINGLE randomized 833 adults to treatment with dolutegravir + abacavir/lamivudine or efavirenz/tenofovir/ emtricitabine. FLAMINGO randomized 484 adults to treatment with dolutegravir or ritonavir-boosted darunavir, each with either abacavir/lamivudine or tenofovir/emtricitabine as selected by the investigator. Furthermore, prespecified statistical lipid analyses for SINGLE were conducted (change from baseline LDL-C and TC). These exploratory analyses were analyses of covariance that adjusted for baseline LDL-C or TC, viral load, and CD4+. Pre-specified analyses were also conducted for FLAMINGO (change from baseline LDL-C, incidence $\geq$ grade 2 LDL-C). The change from baseline LDL-C was analyzed using a repeated measures analysis of covariance, including baseline plasma HIV-1 RNA, background dual NRTI therapy, baseline LDL-C, treatment group, and visit. The incidence of $\geq$ grade 2 LDL-C was analyzed using a Chisquared test.

Data are summarized for the randomized phases of the studies, and for all individuals who received at least one dose of investigational product. Individuals were analyzed according to the treatment they actually received for the majority of time on the study, regardless of randomized treatment group. In each study, fasting plasma samples for lipid measurements were collected at baseline and at weeks $2,4,12,16,32$, and 48 . Variables included in this analysis are TC, LDL-C, HDL-C, TC/HDL ratio, and TG at baseline and week 48. Pooled data of fasting lipid levels from patients assigned to receive dolutegravir in the aforementioned studies are presented as the mean concentration (mg/ $\mathrm{dL}$ ) at baseline and week 48 . We further explored differences in changes from baseline $(\mathrm{mg} / \mathrm{dL})$ to week 48 within each study for dolutegravir and its respective comparators. Clinically significant mean increases from baseline in serum lipids refer to those that would suggest lipid-lowering interventions according to current guidelines [12]. The proportion of patients with Division of AIDS (DAIDS) grade 2-4 elevations in TC, LDL-C, and TG is presented by treatment group. The 2004 DAIDS severe adverse events grading table was used for SPRING-1, while the 2009 version was used for SPRING-2, SINGLE, and FLAMINGO. For individuals who initiated serum lipidlowering agents post-baseline, the last-available fasted, ontreatment lipid values prior to lipid treatment initiation 
were used in place of future, observed on-treatment values. Imputation continued even if the subject discontinued the lipid-lowering agent. On-treatment missing data were not imputed and thus a change from baseline was calculated only for those with baseline and on-treatment values. Individuals receiving lipid-lowering agents at baseline were excluded from the lipid analysis.

\section{Results}

A total of 1,118 subjects were randomized to dolutegravir $50 \mathrm{mg}$ once daily within the four ART-naive studies of the dolutegravir development program. Table 1 describes the baseline demographic characteristics of patients, and specifies the number and percentage of patients co-infected with hepatitis $\mathrm{B}$ and hepatitis $\mathrm{C}$, as well as the percentage of patients who received the backbone co-formulation of abacavir/lamivudine. The median age of patients was between 34 and 40 years. Between 32 and $40 \%$ of patients received abacavir/lamivudine, except in SINGLE, where $100 \%$ of patients who received dolutegravir had abacavir/ lamivudine as the combined backbone.

Table 2 shows pooled lipid data for subjects randomized to dolutegravir $50 \mathrm{mg}$ in four studies: SPRING-1, SPRING-2, SINGLE, and FLAMINGO, including mean serum concentrations of TC, HDL-C, LDL-C, and TG at baseline. Additionally shown are the mean changes in those parameters from baseline to week 48. The proportion of patients experiencing grade $2-4$ elevations in each variable from baseline are provided.

Figure 1 shows box plots with baseline and week 48 plasma concentrations of TC, LDL-C, HDL-C, and TG for those individuals receiving dolutegravir. The week-48 values overlapped with baseline values.

Figure 2 shows the changes from baseline TC at week 48 across the studies, and includes the comparator study- arm values. The changes from baseline TC were comparable between dolutegravir and the comparator treatment groups, with the exception of the SPRING-1 study, in which participants receiving dolutegravir had smaller changes in serum lipids than those receiving efavirenz.

Table 3 shows baseline and changes in mean lipid serum concentrations at 48 weeks and grade $2-4$ elevations in lipids in the SPRING-2 study.

Minor increases in mean TC occurred in both the dolutegravir and raltegravir arms. The mean LDL-C levels showed no clinically significant increases from baseline, with mean values at week 48 within a normal range for both the dolutegravir and raltegravir groups. Similar small increases were seen in mean HDL-C plasma levels in both the dolutegravir and raltegravir arms. The mean TC:HDL ratio slightly declined in both arms. Mean TG levels rose from baseline in both the dolutegravir and raltegravir groups, with elevations being slightly lower in the dolutegravir group, although this difference was not clinically significant. The proportion of patients with grade 2-4 elevations was between 5 and $8 \%$ for TC and LDL-C, and $2 \%$ for TG in all evaluable subjects.

In the SINGLE study (Table 4), baseline TC levels were comparable between subjects receiving dolutegravir or efavirenz. At week 48, those receiving efavirenz showed numerically higher mean TC increases compared with dolutegravir recipients. A pre-specified exploratory analysis of change from baseline showed the difference in increase to be statistically significant $(p=0.005)$. Numerical differences were also observed in LDL-C, with the highest changes from baseline in patients assigned to the efavirenz arm; the pre-specified exploratory analysis of change from baseline showed this difference to be statistically significant $(p=0.032)$. The mean HDL-C plasma levels modestly increased from baseline to week 48 in both the dolutegravir and efavirenz arms. With respect to the TC:HDL ratio, a slight decrease from mean baseline ratio

Table 1 Baseline demographic characteristics of patients included in the studies

\begin{tabular}{|c|c|c|c|c|c|c|c|c|}
\hline \multirow[t]{2}{*}{ Characteristic } & \multicolumn{2}{|l|}{ SPRING-1 } & \multicolumn{2}{|l|}{ SPRING-2 } & \multicolumn{2}{|l|}{ SINGLE } & \multicolumn{2}{|c|}{ FLAMINGO } \\
\hline & $\begin{array}{l}\text { DTG } \\
(N=51)\end{array}$ & $\begin{array}{l}\text { EFV } \\
(N=50)\end{array}$ & $\begin{array}{l}\text { DTG } \\
(N=411)\end{array}$ & $\begin{array}{l}\text { RAL } \\
(N=411)\end{array}$ & $\begin{array}{l}\text { DTG } \\
(N=414)\end{array}$ & $\begin{array}{l}\text { ATR } \\
(N=419)\end{array}$ & $\begin{array}{l}\text { DTG } \\
(N=242)\end{array}$ & $\begin{array}{l}\text { DRV } \\
(N=242)\end{array}$ \\
\hline Median age, years & 37 & 40 & 37 & 35 & 36 & 35 & 34 & 34 \\
\hline Male & $45(88)$ & $44(88)$ & $348(85)$ & $355(86)$ & 347 (84) & $356(85)$ & $211(87)$ & $201(83)$ \\
\hline White & $38(75)$ & $43(86)$ & $346(84)$ & $352(86)$ & $284(69)$ & $284(68)$ & $173(71)$ & $176(73)$ \\
\hline $\begin{array}{l}\text { ABC/3TC NRTI } \\
\text { background }\end{array}$ & $17(33)$ & $16(32)$ & $169(41)$ & $164(40)$ & 414 (100) & 0 & $79(33)$ & $80(33)$ \\
\hline Hepatitis B and/or C & $4(8)$ & $6(12)$ & $49(12)$ & $43(10)$ & $28(7)$ & $30(7)$ & $26(11)$ & $20(8)$ \\
\hline
\end{tabular}

All data are expressed as $n(\%)$ unless otherwise specified

$A B C / 3 T C$ abacavir/lamivudine, $A T R$ atripla, $D R V$ darunavir, $D T G$ dolutegravir, $E F V$ efavirenz, $N R T I$ nucleoside reverse transcriptase inhibitor, $R A L$ raltegravir 
Table 2 Pooled dolutegravir mean changes from baseline of lipid serum levels from SPRING-1, SPRING-2, SINGLE, and FLAMINGO, and maximal post-baseline emergent grade $2-4$ elevations

\begin{tabular}{|c|c|c|c|c|c|}
\hline \multirow[t]{2}{*}{ Parameter } & \multicolumn{2}{|c|}{ Baseline } & \multicolumn{2}{|c|}{ Change from baseline to week 48} & \multirow{2}{*}{$\begin{array}{l}\text { Maximum post-baseline } \\
\text { emergent grade } 2-4 \text { elevations } \\
n / N(\%)\end{array}$} \\
\hline & $n$ & Mean (SD) & $n$ & Mean (SD) & \\
\hline $\mathrm{TC}(\mathrm{mg} / \mathrm{dL})$ & 953 & $160.5(34)$ & 834 & $9.9(27)$ & $78 / 1,118(7)$ \\
\hline LDL-C (mg/dL) & 946 & $94.1(29)$ & 821 & $4.8(21)$ & $65 / 1,118(6)$ \\
\hline Triglycerides (mg/dL) & 954 & $114.7(71)$ & 836 & $8.7(85)$ & $18 / 1,118(2)$ \\
\hline HDL-C (mg/dL) & 953 & $43.9(13)$ & 834 & $3.6(10)$ & NA \\
\hline TC:HDL ratio & 1,013 & $3.9(1)$ & 888 & $-0.1(1)$ & NA \\
\hline
\end{tabular}

DAIDS Division of AIDS, $H D L-C$ high-density lipoprotein cholesterol, $L D L-C$ low-density lipoprotein cholesterol, $N A$ not applicable, $S D$ standard deviation, $T C$ total cholesterol

a DAIDS grading system

a

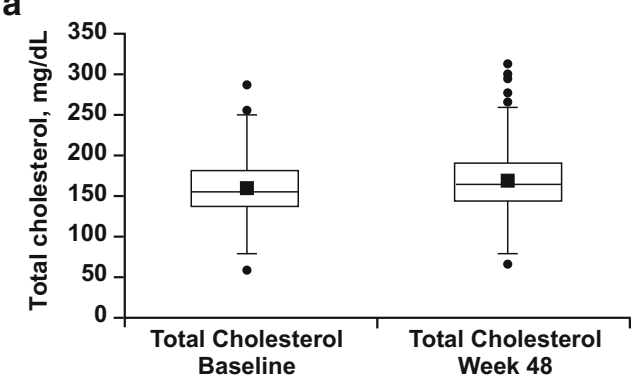

b

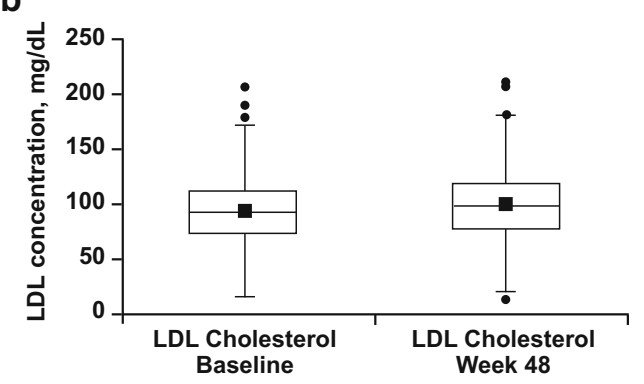

C

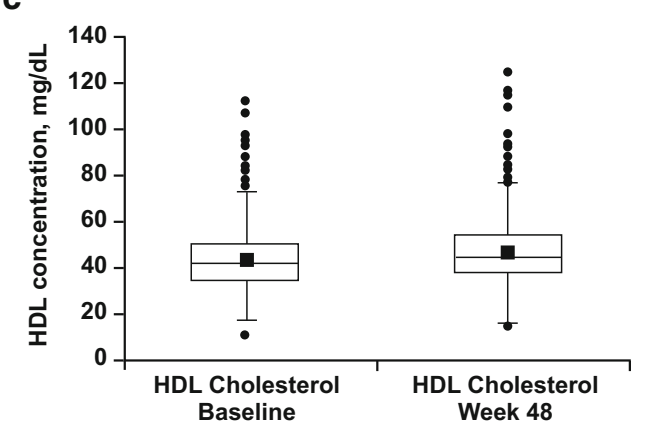

Fig. 1 Pooled DTG at baseline and at week 48 a total cholesterol, b LDL cholesterol, c HDL cholesterol, and d triglyceride levels. Boxes represent the first to third quartile; lines and symbols within the box represent medians and means, respectively. Whiskers extend to

in both the dolutegravir and efavirenz groups was observed, with no differences at week 48 between treatment arms $(-0.1)$. Levels of TG increased in both arms, with no significant difference in the mean change between d

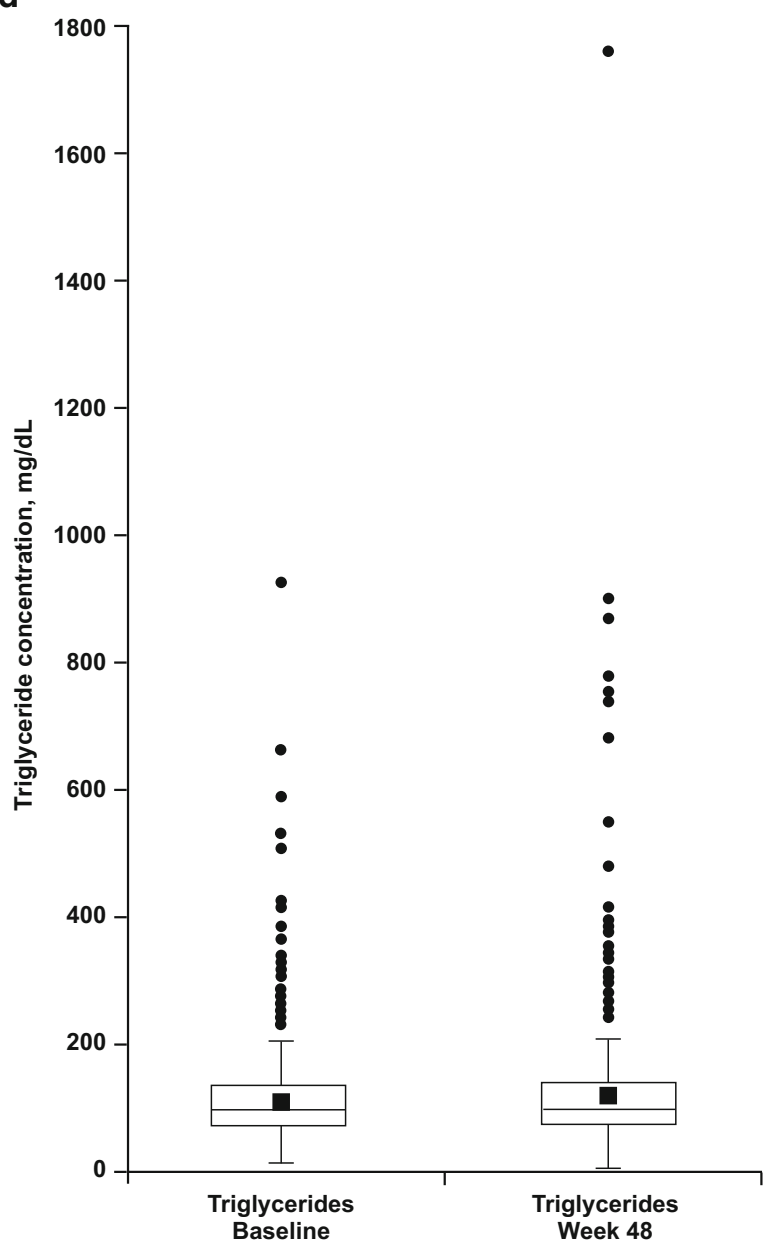

the most extreme observations within 1.5 times the length of the interquartile range from the first and third quartiles. Points beyond the whiskers are considered outliers. DTG dolutegravir, HDL highdensity lipoprotein, $L D L$ low-density lipoprotein

the dolutegravir and efavirenz groups (17.7 vs. $18.6 \mathrm{mg} / \mathrm{dL}$ at week 48 , respectively). The proportion of patients with grade 2-4 elevations was between 6 and $8 \%$ for TC and LDL-C, and $2 \%$ for TG. 


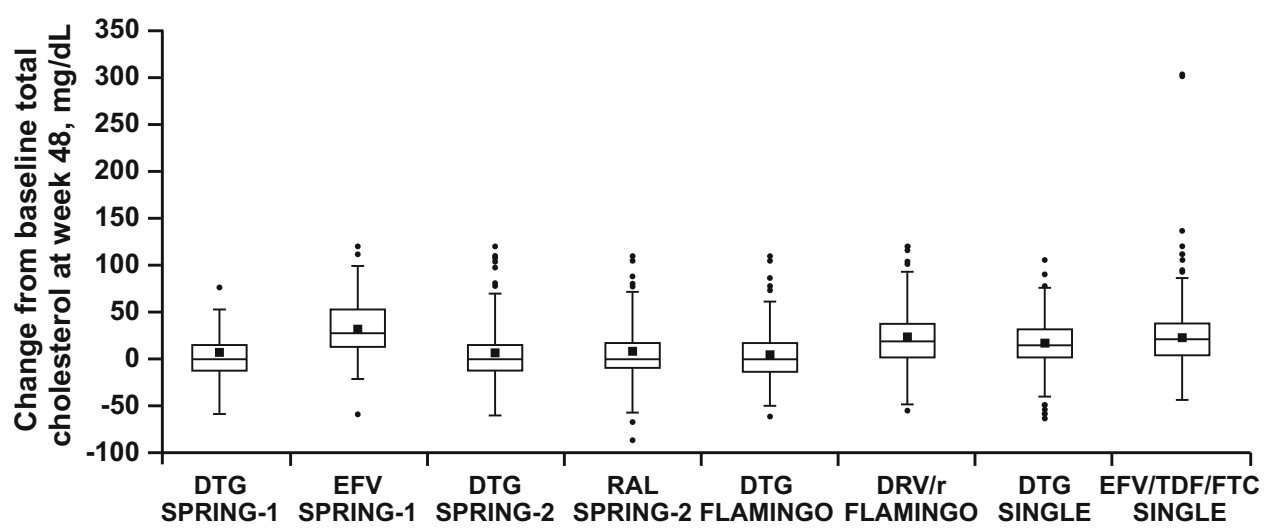

Fig. 2 Changes from baseline total cholesterol at week 48. Boxes represent the first to third quartile; lines and symbols within the box represent medians and means, respectively. Whiskers extend to the most extreme observations within 1.5 times the length of the interquartile range from the first and third quartiles. Points beyond the whiskers are considered outliers. $D R V / r$ darunavir/ritonavir, $D T G$ dolutegravir, $E F V$ efavirenz, $E F V / T D F / F T C$ efavirenz/tenofovir disoproxil fumarate/emtricitabine, $R A L$ raltegravir

Table 3 Mean lipid changes from baseline to week 48 in the SPRING-2 study

\begin{tabular}{|c|c|c|c|c|c|c|}
\hline \multirow[t]{2}{*}{ Parameter } & \multirow[t]{2}{*}{ Treatment } & \multicolumn{2}{|c|}{ Baseline } & \multicolumn{2}{|c|}{ Change from baseline } & \multirow{2}{*}{$\begin{array}{l}\text { Maximum post-baseline } \\
\text { emergent grade } 2-4 \text { elevations } \\
n / N(\%)\end{array}$} \\
\hline & & $n$ & Mean (SD) & $n$ & Mean (SD) & \\
\hline \multirow[t]{2}{*}{$\mathrm{TC}(\mathrm{mg} / \mathrm{dL})$} & DTG & 356 & $163.8(34)$ & 318 & $6.9(28)$ & $32 / 411(8)$ \\
\hline & RAL & 366 & $160.3(38)$ & 308 & $9.0(29)$ & 24/411 (6) \\
\hline \multirow[t]{2}{*}{ LDL-C (mg/dL) } & DTG & 354 & $96.8(30)$ & 313 & $2.9(21)$ & 29/411 (5) \\
\hline & RAL & 363 & $93.4(32)$ & 302 & $3.3(23)$ & 22/411 (5) \\
\hline \multirow[t]{2}{*}{ HDL-C (mg/dL) } & DTG & 356 & $44.4(12)$ & 318 & $2.7(11)$ & NA \\
\hline & RAL & 366 & $44.3(13)$ & 308 & $2.7(10)$ & NA \\
\hline \multirow[t]{2}{*}{ TC:HDL ratio } & DTG & 356 & $3.9(1)$ & 318 & $-0.04(1)$ & NA \\
\hline & RAL & 366 & $3.8(1)$ & 308 & $-0.1(2)$ & NA \\
\hline \multirow[t]{2}{*}{ Triglycerides (mg/dL) } & DTG & 357 & $113.8(64)$ & 319 & $8.6(91)$ & 7/411 (2) \\
\hline & RAL & 366 & 115.9 (82) & 308 & $10.1(93)$ & 8/411 (2) \\
\hline
\end{tabular}

DAIDS Division of AIDS, DTG dolutegravir, $H D L-C$ high-density lipoprotein cholesterol, $L D L-C$ low-density lipoprotein cholesterol, $N A$ not applicable, $R A L$ raltegravir, $S D$ standard deviation, $T C$ total cholesterol

${ }^{a}$ DAIDS grading system

Figure 3 shows box plots with mean changes from baseline to week 48 in lipid parameters across the four comparative studies of dolutegravir versus raltegravir (SPRING-1, SPRING-2), efavirenz (SINGLE), and ritonavir-boosted darunavir (FLAMINGO), respectively.

Table 5 shows lipid changes in the FLAMINGO study. The mean increase in TC in the ritonavir-boosted darunavir arm from baseline to 48 weeks was clinically significantly greater in the ritonavir-boosted darunavir arm; a greater mean increase in LDL-C was also observed in the ritonavir-boosted darunavir arm. In the FLAMINGO study, a pre-specified analysis of the mean change from baseline to week 48 in LDL-C demonstrated a significantly lower $(p<0.001)$ LDL-C increase in the dolutegravir group than in the ritonavir-boosted darunavir group. The mean increase in HDL-C levels from baseline to week 48 was small and comparable between dolutegravir and ritonavir-boosted darunavir. With regard to TC:HDL ratios, the dolutegravir arm showed no increase in the TC:HDL ratio versus an increase of 0.4 in the ritonavir-boosted darunavir arm from baseline to week 48. The mean changes in TG levels increased in the ritonavir-boosted darunavir group and decreased in the dolutegravir group.

Grade 2-4 maximum post-baseline toxicities for dolutegravir and ritonavir-boosted darunavir for $\mathrm{TC}$ and $\mathrm{TG}$ were reported in 4 versus $16 \%$ and $<1 \%$ versus $3 \%$ of subjects, respectively. A similar difference was shown for these reports with LDL-C (4\% for subjects receiving dolutegravir vs. $14 \%$ for those receiving ritonavir-boosted darunavir). A pre-specified analysis showed the dolutegravir group had significantly fewer LDL values of grade 2 or higher than the ritonavir-boosted darunavir group $(p<0.001)$ [9]. 
Table 4 Mean lipid changes from baseline to week 48 in the SINGLE study

\begin{tabular}{|c|c|c|c|c|c|c|}
\hline \multirow[t]{2}{*}{ Parameter } & \multirow[t]{2}{*}{ Treatment } & \multicolumn{2}{|c|}{ Baseline } & \multicolumn{2}{|c|}{ Change from baseline } & \multirow{2}{*}{$\begin{array}{l}\text { Maximum post-baseline } \\
\text { emergent grade } 2-4 \text { elevations } \\
n / N(\%)\end{array}$} \\
\hline & & $n$ & Mean (SD) & $n$ & Mean (SD) & \\
\hline \multirow[t]{2}{*}{$\mathrm{TC}(\mathrm{mg} / \mathrm{dL})$} & DTG & 346 & $158.9(34)$ & 296 & $17.1(26)$ & $35 / 414(8)$ \\
\hline & EFV & 355 & $158.2(37)$ & 279 & $24.1(34)$ & $32 / 419(8)$ \\
\hline \multirow[t]{2}{*}{ LDL-C (mg/dL) } & DTG & 342 & $93.1(29)$ & 289 & $8.5(21)$ & $32 / 414(8)$ \\
\hline & EFV & 353 & $92.7(22)$ & 273 & $13.1(30)$ & $24 / 419(6)$ \\
\hline \multirow[t]{2}{*}{ HDL-C (mg/dL) } & DTG & 346 & $43.4(13)$ & 296 & $5.2(9)$ & NA \\
\hline & EFV & 355 & $43.6(13)$ & 279 & $8.0(11)$ & NA \\
\hline \multirow[t]{2}{*}{ TC:HDL ratio } & DTG & 374 & $3.9(1)$ & 320 & $-0.1(1)$ & NA \\
\hline & EFV & 381 & $3.9(1)$ & 302 & $-0.1(1)$ & NA \\
\hline \multirow[t]{2}{*}{ Triglycerides (mg/dL) } & DTG & 346 & $115.0(78)$ & 296 & $17.7(94)$ & $8 / 414(2)$ \\
\hline & EFV & 355 & $111.2(67)$ & 279 & $18.6(92)$ & 9/419 (2) \\
\hline
\end{tabular}

DAIDS Division of AIDS, DTG dolutegravir, $E F V$ efavirenz, $H D L-C$ high-density lipoprotein cholesterol, $L D L-C$ low-density lipoprotein cholesterol, $N A$ not applicable, $S D$ standard deviation, $T C$ total cholesterol

a DAIDS grading system

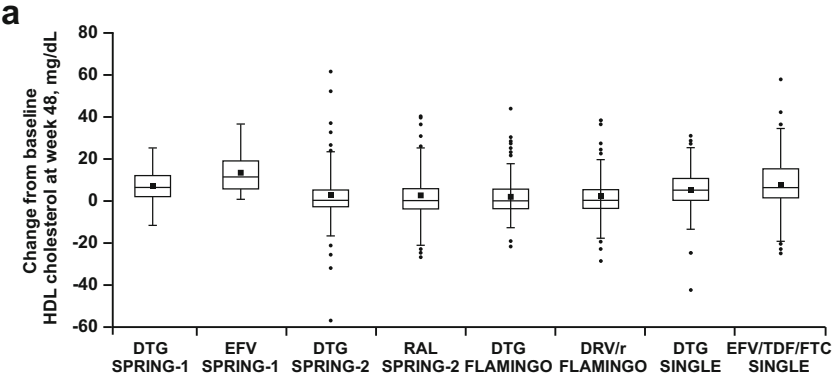

c

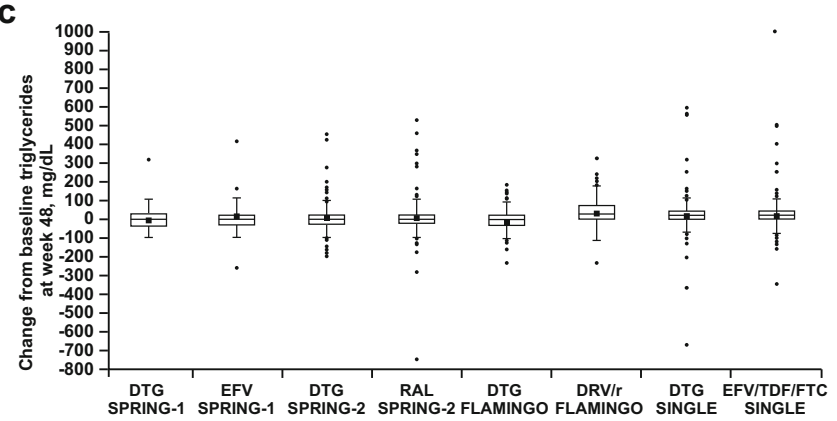

Fig. 3 Changes from baseline in a HDL cholesterol, b LDL cholesterol, $\mathbf{c}$ triglycerides, and $\mathbf{d}$ total cholesterol/HDL ratio at 48 weeks. Boxes represent the first to third quartile; lines and symbols within the box represent medians and means, respectively. Whiskers extend to the most extreme observations within 1.5 times the length of the

\section{Discussion}

This analysis provides information on lipid outcomes at 48 weeks from phase IIb and III controlled studies within the dolutegravir development programme, and allows for a comparison of the effect on lipids of dolutegravir versus other classes of treatments for HIV, including non-NRTIs (NNRTIs; efavirenz), boosted protease inhibitors b

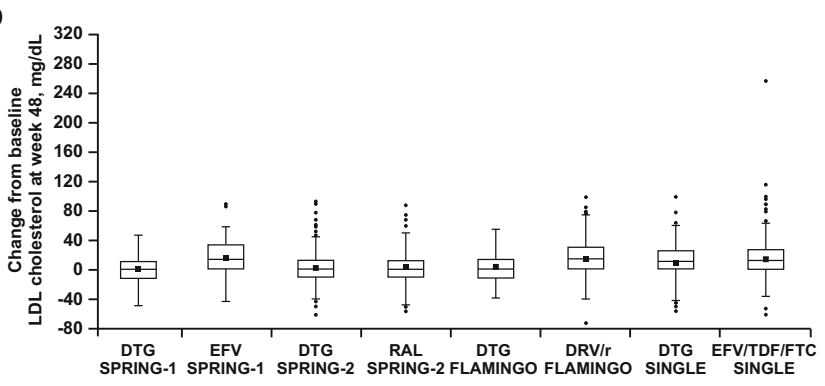

d

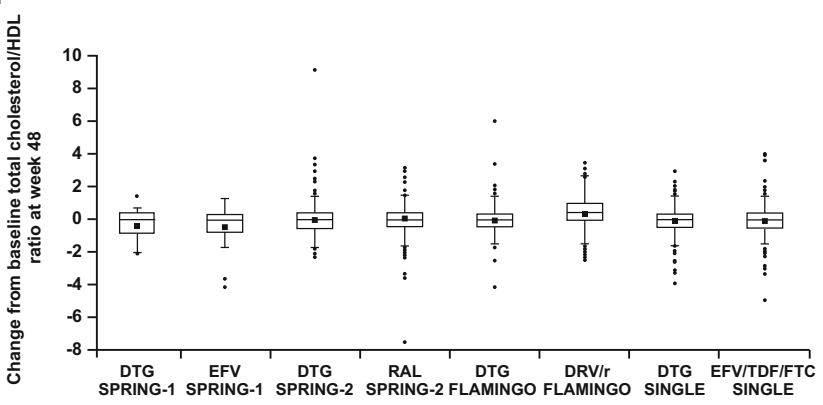

interquartile range from the first and third quartiles. Points beyond the whiskers are considered outliers. DRV/r darunavir/ritonavir, DTG dolutegravir, $E F V$ efavirenz, EFV/TDF/FTC efavirenz/tenofovir disoproxil fumarate/emtricitabine, $H D L$ high-density lipoprotein, $L D L$ low-density lipoprotein, $R A L$ raltegravir

(ritonavir-boosted darunavir), and a first-generation INSTI, raltegravir. Our data confirm initial observations suggesting a minimal effect of dolutegravir in combination with NRTIs on serum lipids in ART-naive subjects infected with HIV [11]. Dolutegravir also demonstrated an effect on serum lipids that was smaller than that observed with efavirenz or boosted darunavir, regardless of the choice of NRTI backbone. 
Table 5 Mean lipid changes from baseline to week 48 in the FLAMINGO study

\begin{tabular}{|c|c|c|c|c|c|c|}
\hline \multirow[t]{2}{*}{ Parameter } & \multirow[t]{2}{*}{ Treatment } & \multicolumn{2}{|c|}{ Baseline } & \multicolumn{2}{|c|}{ Change from baseline } & \multirow{2}{*}{$\begin{array}{l}\text { Maximum post-baseline } \\
\text { emergent grade } 3-4^{\mathrm{a}} \\
n / N(\%)\end{array}$} \\
\hline & & $n$ & Mean (SD) & $n$ & Mean (SD) & \\
\hline \multirow[t]{2}{*}{$\mathrm{TC}(\mathrm{mg} / \mathrm{dL})$} & DTG & 207 & $157.6(33)$ & 182 & $4.3(24)$ & $9 / 242(4)$ \\
\hline & $\mathrm{DRV} / \mathrm{r}$ & 215 & $162.5(35)$ & 178 & $22.5(33)$ & $39 / 242(16)$ \\
\hline \multirow[t]{2}{*}{ LDL-C (mg/dL) } & DTG & 207 & $91.1(29)$ & 182 & $3.1(20)$ & $10 / 242(4)$ \\
\hline & $\mathrm{DRV} / \mathrm{r}$ & 215 & $95.5(29)$ & 178 & $14.1(25)$ & $35 / 242(14)$ \\
\hline \multirow[t]{2}{*}{ HDL-C (mg/dL) } & DTG & 207 & $43.9(13)$ & 182 & $2.0(9)$ & NA \\
\hline & $\mathrm{DRV} / \mathrm{r}$ & 215 & $43.5(13)$ & 178 & $2.2(10)$ & NA \\
\hline \multirow[t]{2}{*}{ TC:HDL ratio } & DTG & 222 & $3.9(1)$ & 197 & $0.0(1)$ & NA \\
\hline & $\mathrm{DRV} / \mathrm{r}$ & 227 & $4.1(2)$ & 188 & $0.4(1)$ & NA \\
\hline \multirow[t]{2}{*}{ Triglycerides (mg/dL) } & DTG & 207 & $114.0(66)$ & 183 & $-5.5(53)$ & $2 / 242(<1)$ \\
\hline & $\mathrm{DRV} / \mathrm{r}$ & 215 & $117.9(67)$ & 178 & $33.1(73)$ & $8 / 242(3)$ \\
\hline
\end{tabular}

DAIDS Division of AIDS, $D R V / r$ darunavir/ritonavir, $D T G$ dolutegravir, $H D L-C$ high-density lipoprotein cholesterol, $L D L-C$ low-density lipoprotein cholesterol, $N A$ not applicable, $S D$ standard deviation, $T C$ total cholesterol

${ }^{\text {a }}$ DAIDS grading system

In individuals receiving dolutegravir, levels of LDL-C, a primary target of lipid-lowering therapies, minimally increased to a mean concentration of $99 \mathrm{mg} / \mathrm{dL}$ at 48 weeks across the four clinical studies. This is just below the $100 \mathrm{mg} / \mathrm{dL}$ target previously recommended by the National Cholesterol Education Program (NCEP) guidelines for subjects with the highest risk of cardiovascular disease [13]. On the other hand, increased levels of HDL$\mathrm{C}$, which are associated with decreased risk of coronary artery disease, rose from $43 \mathrm{mg} / \mathrm{dL}$ at baseline to $47 \mathrm{mg} / \mathrm{dL}$ at 48 weeks. Furthermore, a mean change from baseline in TC:HDL ratio of -0.1 , which infers a low risk of cardiovascular disease, characterized the dolutegravir arms.

With respect to hypertriglyceridemia, which is considered an independent risk factor for cardiovascular disease, individuals exposed to dolutegravir showed modest mean increases in TG levels (from 114 to $122 \mathrm{mg} / \mathrm{dL}$ ), which were below the $200 \mathrm{mg} / \mathrm{dL}$ threshold used for recommendation of therapy ('borderline high' according to 2001 NCEP guidelines) [14]. All of the above suggest a convenient lipid profile of dolutegravir in ART-naive subjects based on regimens including dolutegravir during 48 weeks of combination therapy.

In the comparative analysis, similar lipid effects were observed between the dolutegravir and raltegravir regimens. Results from the SPRING-2 study showed minor increases in TC, LDL-C, and TG levels in both arms without reaching clinically significant concentrations at 48 weeks. The mean lipid changes observed in the raltegravir arm were consistent with those previously reported in the STARTMRK study, in which raltegravir had minimal effects on lipid levels in ART-naive subjects [15]. Together, these results confirm the previous results reported regarding the equivalent effect on lipids of the INSTI drug class.

In the SINGLE study, there were larger mean increases in TC, LDL-C, HDL-C, and TG in patients randomized to efavirenz compared with those randomized to dolutegravir. Similar effects on lipids were reported in efavirenztreated patients participating in several previous clinical trials [16]. Of note, a larger increase of HDL-C in efavirenz subjects probably balanced the mean TC:HDL ratio between efavirenz and dolutegravir patients in the current analysis.

In the FLAMINGO study, greater increases in TC, LDL$\mathrm{C}$, and TG levels were observed in the ritonavir-boosted darunavir arm. This is consistent with the effect of drugs in the protease inhibitor (PI) class on serum lipids [17]. However, it should be noted that different antiretroviral drugs within the same class may induce differential effects on lipids. As an example, across a number of clinical studies, atazanavir in combination with NRTIs has shown consistently smaller increases in triglycerides and cholesterol than combinations including lopinavir/ritonavir [18]. Nonetheless, it should be noted that a significant proportion of the lipid effect of the PI-based regimens is related to the concomitant use of the booster ritonavir with a PI. Noteworthy, in contrast with elvitegravir and protease inhibitors, the pharmacokinetic characteristics of dolutegravir allow for its use without a pharmacological booster.

Standard measurements of cholesterol and triglycerides may not fully reflect the complex changes in lipid metabolism induced by HIV and highly active ART. Other markers of cardiovascular risk, including apolipoproteins, d-dimer, and non-invasive imaging tools among others, have been shown to provide additional information to 
predict a cardiovascular event. Nevertheless, traditional risk factors such as increased cholesterol serum levels remain significantly associated with a high risk of a cardiovascular event and provide practical information for clinical management of HIV-infected individuals [12].

The specific effect of background nucleosides on lipids is not reported in this study. Nevertheless, it has been reported that NRTIs, alone or co-formulated, could have effects on lipid levels and could even have lipid-lowering effects [19-22]. In this analysis, the distribution of the two backbone options was balanced and comparable between arms, ensuring an accurate interpretation of the lipid profile of dolutegravir, except in the SINGLE study, where all patients received dolutegravir with abacavir/lamivudine in the dolutegravir arm versus the efavirenz arm, where all patients received tenofovir/emtricitabine. We can then hypothesize that the lipid-lowering effect of tenofovir might counterbalance the lipid effect of efavirenz versus the neutral lipid profile of dolutegravir.

Additional analysis of these and other studies, as well as results from NRTI-sparing regimens, would provide additional information on the impact of NRTIs on lipid levels in the context of combination therapy.

\section{Conclusions}

A broadly neutral lipid effect, as shown in this analysis, adds to the strength of dolutegravir as an initial therapy for the treatment of HIV. This is of particular importance for an aging population with other risk factors for metabolic syndrome and cardiovascular disease [23]. The data presented are limited to 48 weeks of exposure to antiretrovirals within the context of clinical trials. An extended follow-up and expanded use of dolutegravir in clinical practice should help to further characterize its lipid profile.

Acknowledgments Funding for this work was provided by ViiV Healthcare. Romina Quercia, Jeremy Roberts, Louise Martin-Carpenter, and Carlos Zala meet the criteria for authorship set forth by the International Committee for Medical Journal Editors. The authors wish to acknowledge Mike Hast and Clint Smith for editorial assistance during the development of this manuscript.

Conflicts of interest Romina Quercia and Louise Martin-Carpenter are employees of ViiV Healthcare. Jeremy Roberts is an employee of GlaxoSmithKline and has received support from GlaxoSmithKline for work outside of the submitted work. Carlos Zala declares no conflicts of interest.

All authors have full control of all primary data and agree to allow the journal to review the data if requested.

Open Access This article is distributed under the terms of the Creative Commons Attribution Noncommercial License which permits any noncommercial use, distribution, and reproduction in any medium, provided the original author(s) and the source are credited.

\section{References}

1. Cohan GR. HIV-associated metabolic and morphologic abnormality syndrome: welcome therapy may have unwelcome effects. Postgrad Med. 2000;107:141-6.

2. Feeney ER, Mallon PW. HIV and HAART-associated dyslipidemia. Open Cardiovasc Med J. 2011;5:49-63.

3. Grunfeld C. Dyslipidemia and its treatment in HIV infection. Top HIV Med. 2010;18:112-8.

4. Friis-Møller N, Weber R, Reiss P, Thiébaut R, Kirk O, d'Arminio Monforte A, et al. Cardiovascular disease risk factors in HIV patients: association with antiretroviral therapy. Results from the DAD study. AIDS. 2003;17:1179-93.

5. Raffi F, Jaeger H, Quiros-Roldan E, Albrecht H, Belonosova E, Gatell JM, Extended SPRING-2 study group, et al. Once-daily dolutegravir versus twice-daily raltegravir in antiretroviral-naive adults with HIV-1 infection (SPRING-2 study): 96 week results from a randomised, double-blind, non-inferiority trial. Lancet Infect Dis. 2013;13:927-35.

6. Raffi F, Rachlis A, Stellbrink H-J, Hardy WD, Torti C, Orkin C, et al. Once-daily dolutegravir versus raltegravir in antiretroviralnaive adults with HIV-1 infection: 48 week results from the randomised, double-blind, non-inferiority SPRING-2 study. Lancet. 2013;381:735-43.

7. Cahn P, Pozniak AL, Mingrone H, Shuldyakov A, Brites C, Andrade-Villanueva JF, Extended SAILING study team, et al. Dolutegravir versus raltegravir in antiretroviral-experienced, integrase-inhibitor-naive adults with HIV: week 48 results from the randomised, double-blind, non-inferiority SAILING study. Lancet. 2013;382:700-8.

8. Walmsley SL, Antela A, Clumeck N, Duiculescu D, Eberhard A, Gutiérrez F, SINGLE investigators, et al. Dolutegravir plus abacavir-lamivudine for the treatment of HIV-1 infection. N Engl J Med. 2013;369:1807-18.

9. Clotet B, Feinberg J, van Lunzen J, Khuong-Josses MA, Antinori A, Dumitru I, ING114915 study team, et al. Once-daily dolutegravir versus darunavir plus ritonavir in antiretroviral-naive adults with HIV-1 infection (FLAMINGO): 48 week results from the randomised open-label phase $3 \mathrm{~b}$ study. Lancet. 2014;383:2222-31.

10. US Department of Health and Human Services Panel on Antiretroviral Guidelines for Adults and Adolescents. Recommendation on integrase inhibitor use in antiretroviral treatment-naive HIV-infected individuals from the HHS panel on antiretroviral guidelines for adults and adolescents. 2013. Available at: http:// aidsinfo.nih.gov/contentfiles/upload/adultarv_instirecommenda tions.pdf. Accessed 1 Nov 2013.

11. van Lunzen J, Maggiolo F, Arribas JR, Rakhmanova A, Yeni P, Young B, et al. Once daily dolutegravir (S/GSK1349572) in combination therapy in antiretroviral-naive adults with HIV: planned interim 48 week results from SPRING-1, a dose-ranging, randomised, phase $2 b$ trial. Lancet Infect Dis. 2012;12:111-8.

12. Dubé MP, Stein JH, Aberg JA, Fichtenbaum CJ, Gerber JG, Tashima KT, Adult AIDS Clinical Trial Group Cardiovascular Subcommittee and the HIV Medical Association of the Infectious Disease Society of America, et al. Guidelines for the evaluation and management of dyslipidemia in human immunodeficiency virus (HIV)-infected adults receiving antiretroviral therapy: recommendations of the HIV Medical Association of the Infectious Disease Society of America and the Adult AIDS Clinical Trials Group. Clin Infect Dis. 2003;37:613-27.

13. Stone NJ, Robinson JG, Lichtenstein AH, Bairey Merz CN, Blum $\mathrm{CB}$, Eckel RH, et al. 2013 ACC/AHA guideline on the treatment of blood cholesterol to reduce atherosclerotic cardiovascular risk in adults: a report of the American College of Cardiology/ 
American Heart Association Task Force on Practice Guidelines. Circulation. 2014;129(25 Suppl 2):S1-45.

14. Miller M, Stone NJ, Ballantyne C, Bittner V, Criqui MH, Ginsberg HN, et al. Triglycerides and cardiovascular disease: a scientific statement from the American Heart Association. Circulation. 2011;123:2292-333.

15. Rockstroh JK, Lennox JL, Dejesus E, Saag MS, Lazzarin A, Wan $\mathrm{H}$, et al. Long-term treatment with raltegravir or efavirenz combined with tenofovir/emtricitabine for treatment-naive human immunodeficiency virus-1-infected patients: 156-week results from STARTMRK. Clin Infect Dis. 2011;53:807-16.

16. Kirchner JT. A tolerability review of non-nucleoside reverse transcriptase inhibitors: focus on laboratory measures of clinical relevance. J Antivir Antiretrovir. 2012;4:94-100.

17. DAD Study Group. Class of antiretroviral drugs and the risk of myocardial infarction. N Engl J Med. 2007;356:1723-35.

18. Carey D, Amin J, Boyd M, Petoumenos K, Emery S. Lipid profiles in HIV-infected adults receiving atazanavir and atazanavir/ritonavir: systematic review and meta-analysis of randomized controlled trials. J Antimicrob Chemother. 2010;65: 1878-88.
19. Sax P, Tierney C, Collier AC, Daar ES, Mollan K. Abacavirlamivudine versus tenofovir-emtricitabine. $\mathrm{N}$ Engl $\mathrm{J}$ Med. 2009;361:2230-40.

20. Gallant JE, DeJesus E, Arribas JR, Pozniak AL, Gazzard B, Campo RE, Study 934 Group, et al. Tenofovir DF, emtricitabine, and efavirenz vs. zidovudine, lamivudine, and efavirenz for HIV. N Engl J Med. 2006;354:251-60.

21. Campo R, DeJesus E, Bredeek UF, Henry K, Khanlou H, Logue K, et al. SWIFT: prospective 48-week study to evaluate safety of switching to emtricitabine/tenofovir from lamivudine/abacavir in virologically suppressed HIV-1 infected patients on a boosted protease inhibitor containing antiretroviral regimen. Clin Infect Dis. 2013;56:1637-45.

22. Santos JR, Saumoy M, Curran A, Bravo I, Navarro J, Estany C, et al. Randomized, crossover, double-blind, placebo-controlled trial to assess the lipid lowering effect of co-formulated TDF/ FTC. J Int AIDS Soc. 2014;17:19550.

23. Guaraldi G, Orlando G, Zona S, Menozzi M, Carli F, Garlassi E, et al. Premature age-related comorbidities among HIV-infected persons compared with the general population. Clin Infect Dis. 2011;11:1120-6. 